\title{
Improvement of the automated seismic and acoustic monitoring system "Prognoz-ADS" main elements
}

\author{
Pavel Anikin ${ }^{1, *}$, Gennady Kursakin ${ }^{1}$ and Iuliia Fedotova ${ }^{1}$ \\ ${ }^{1}$ Mining Institute FEB RAS, 51 Turgenev st., Khabarovsk, 680000, Russia
}

\begin{abstract}
The results of theoretical and experimental studies have established the need to improve and modernize the highly sensitive piezoelectric resonant type PeA12 converters used in the seismic monitoring system, which have been successfully used for more than 30 years in the rock mass at a number of rockburst hazardous mines. The main problem of the accuracy measurement by the geophone PeA12 (and other models based on it) is due to the presence of several resonances in the operating frequency band. The developed upgraded model of the AP2088 converter has successfully passed industrial testing as part of the automated monitoring system "Prognoz-ADS" at rockburst hazardous mine. During the test period (more than 1 year), seismoacoustic events were registered and verified in the rock mass, including rock burst and shocks in the rock mass. Thus, the use of highly sensitive $\left(\sim 10 \mathrm{~V} / \mathrm{m}^{\circ} \mathrm{s}^{-2}\right)$ piezoacoustic converters AP2088 as part of the automated system provides registration of acoustic emission in the rock mass in the frequency range from 0.1 to $10 \mathrm{kHz}$ with the energy of seismic events from 10 to $10^{6} \mathrm{~J}$, which will increase the reliability of the forecast of geodynamic phenomena and technogenic seismicity in the control zone of the system.
\end{abstract}

\section{Introduction}

When underground mining of deposits in difficult mining and geological conditions, problems associated with increased rock pressure and dynamic manifestations in the form of shocks, shootings, rockbursts and mining-induced seismicity inevitably arise. In the world mining practice, automated seismoacoustic systems are used for monitoring dangerous dynamic phenomena, which allow monitoring physical and geomechanical processes in the rock mass, those that include a network of sensors, also called acoustic emission transducers, piezodetectors or geophones. Geophones register seismoacoustic signals in the frequency range from $100 \mathrm{~Hz}$ to $1-2 \mathrm{kHz}$, which allows us to obtain data on potentially dangerous foci of dynamic phenomena (seismoacoustic events) with energy from $10^{3}$ to $10^{6} \mathrm{~J}$. The acceleration sensitivity of most used seismodetectors is from 0.05 to $0.5 \mathrm{~V} / \mathrm{m} \cdot \mathrm{s}^{-2}$, which is not enough to register high-frequency acoustic signals $(3-10 \mathrm{kHz})$ with an energy of up to $10^{2} \mathrm{~J}$ at a distance of about $80-120 \mathrm{~m}$ from the foci that are

\footnotetext{
*Corresponding author: pav.anik@mail.ru
} 
precursors of large seismoacoustic events in the rock mass. This is why it is important to develop and use highly sensitive geophones with a wide operating frequency band as part of monitoring systems.

In a number of rockburst hazardous deposits in the Russian Federation, regime monitoring of the geomechanical state of rock mass is performed using automated seismicacoustic systems "Prognoz-ADS" developed at the IGD FEB RAS [1-10]. The operation of this system is based on the use of data recorded by an underground network of highly sensitive geophones $\left(8 \mathrm{~V} / \mathrm{m}^{-2} \mathrm{~s}^{-2}\right)$ in the frequency range from 0.2 to $10 \mathrm{kHz}$ with an event energy of 10 to $10^{6} \mathrm{~J}$. During monitoring and a number of accompanying studies, it was established that the first pulses of acoustic emission from rock destruction at the initial stage of stress concentration zones (potential foci of dynamic phenomena) formation are the most important information for predicting dangerous geodynamic phenomena in the rock mass of the mine field and at its closest borders. Such acoustic emission signals are registered in the frequency range from 3 to $10 \mathrm{kHz}$ and higher. Therefore, the characteristics of geophones for monitoring systems should be given special attention.

\section{The geophones characteristics}

The object of research is highly sensitive geophones of the "Prognoz-ADS" system. Since the installation of the system was used geophones (piezoelectric accelerometers) PeA12 developed between 1975-1982 which were issued to SIA "Dalstandart" and until 1993. Since 2003 the manufacture of geophones resumed in the IGD FEB RAS (KhFIC FEB RAS, Khabarovsk). For the first time, PeA12 geophones were used in 1986 in the "Prognoz-5" seismic monitoring system at the Tashtagolsky rockburst hazardous mine. Later they were used as part of the "Prognoz-5" and "Gorizont-6" systems in 1989 at the Tyymyrsky mine, in 1993 as part of the "Prognoz-5AM" monitoring system at the Khingansky mine, and since 2003 at the Nikolayevsky mine. These were analog systems for monitoring rock pressure. PeA12 converters were used in the digital automated system "Prognoz-ADS" installed at rockburst hazardous underground mines: in 2005-the uranium "No.1" of PIMCU PJSC; in 2011 - the polymetallic "Nikolaevsky" of JSC "MMC Dalpolymetal"; in 2015 - the diamond "Mir" of AK"ALROSA" and the apatite-nepheline "United Kirovsky" KB of JSC "Apatit". During the use of geophones, they have shown sufficient reliability and high sensitivity when registering acoustic emission pulses at the initial stage of changing the geomechanical state of rocks.

Structurally, the body of the cylindrical geophone $(\varnothing=65 \mathrm{~mm})$ is made of $20 \mathrm{X} 13$ stainless steel, its mass in the assembly is $1050 \mathrm{~g}$. In the case between the base and the inertial platform, there are two piezoelectric elements glued together $(30 \mathrm{~mm}$ dimeter, $12 \mathrm{~mm}$ high, with two electrodes) that convert elastic vibrations into an electrical signal, figure 1. The output of the integrated amplifier forms a symmetrical electrical signal. To pair the geophone with a rock mass, an embedded part is used, which is cemented in the bottom of horizontal wells 2-3 m long. Main technical characteristics of the PeA12 converter: operating frequency range $0.2 \ldots 10 \mathrm{kHz}$; axial sensitivity for recording acoustic emission in an array at a distance of up to $120 \mathrm{~m}$ - from 0.2 to $7.5 \mathrm{~V} / \mathrm{m}^{\bullet} \mathrm{s}^{-2}$ (resonant type); resistance to mechanical shock $-5 \cdot 10^{3} \mathrm{~g}$; dynamic range of amplitude measurement - up to $30 \mathrm{~dB}$; supply voltage-3.3 V; maximum output voltage - up to $50 \mathrm{mV}$; overall dimensions: length $90 \mathrm{~mm}$, diameter $65 \mathrm{~mm}$. The type of frequency response (frequency response) of the converter is shown in figure 2. During the use of piezoelectric converters PeA12 obtained experience in their application for recording acoustic emission signals in a rock mass at a distance of up to $100 \mathrm{~m}$ in the frequency range of $0.2-10 \mathrm{kHz}$, which is achieved by a relatively high sensitivity at 4 peaks of resonances. 


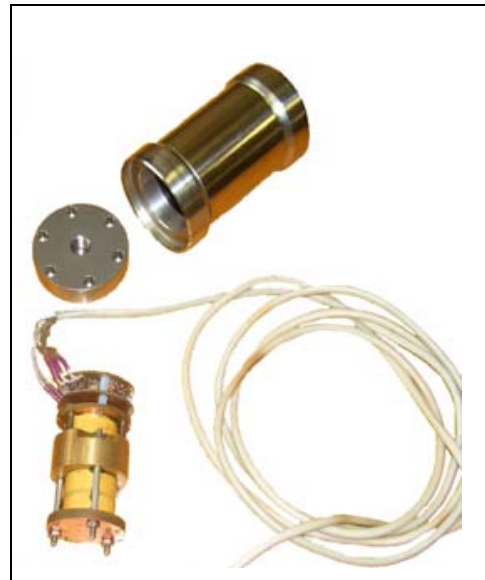

a)

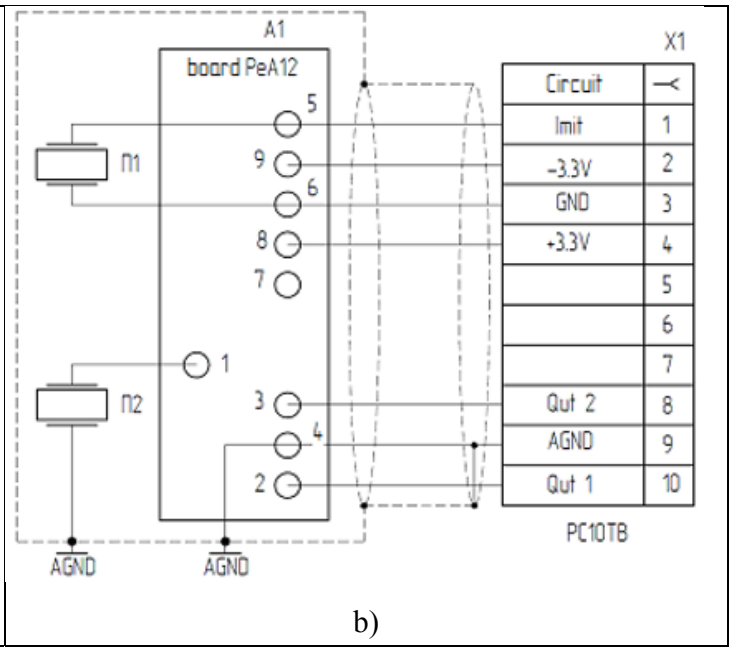

Fig. 1. Geophone PeA12: a) - general view; b) schematic diagram of the converter

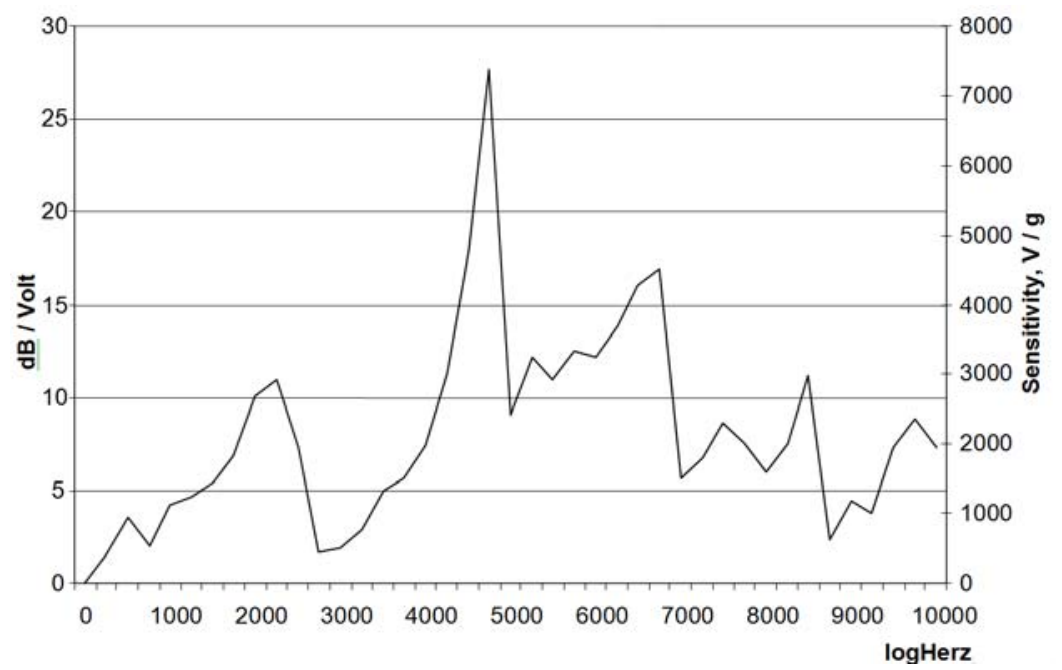

Fig. 2. Amplitude-frequency characteristics of the PeA12

\section{The geophones modernization}

Research in the field of forecasting dangerous geodynamic phenomena shows that it is not enough to obtain only the time characteristics of the signal (with an accuracy of $0.02 \mathrm{~ms}$ ) and calculate the coordinates of the focus (with an error of up to 3-5 m). Based on the results obtained, the main directions of further modernization of geophones and systems themselves were established to improve the quality of analysis of changing processes of geomechanical state of rocks. In particular: to increase the sensitivity of geophones; to measure the amplitude-frequency characteristics of acoustic emission signals with a sufficient degree of reliability; perform frequency analysis; calculate event parameters (energy of seismoacoustic events).

In 2018-2019, PeA12 was modernized and a new geophone model was developed in the following direction: changing the design of the converter (material, mass, piezoceramic model); increasing the stability of the frequency response of the converter $( \pm 10 \%)$ in the 
entire operating frequency range (from 0.2 to $10 \mathrm{kHz}$ ); increasing the axial acceleration sensitivity to $10 \mathrm{~V} / \mathrm{m}^{\circ} \mathrm{s}^{-2}$; increasing the dynamic range of amplitude measurement to $50 \mathrm{~dB}$; conducting industrial tests of new models; certification in the industry certification bodies of the new measuring tool. As a result of modernization of the PeA12 prototype, experimental models PeA12K, AP2088 and AP2088A were developed.

The PeA12K model has the same electronic and piezoelectric components as the PeA12, as well as similar technical and resonant frequency response. There are also 4 resonance peaks in the operating frequency range. Structurally, the body of the geophone is a combined polymer material "Kaprolon" of the b brand and steel of the $20 \mathrm{X} 13$ brand. The contact area of the sensor is made of 20X13 steel and is connected to the polymer housing by a bolted connection. The weight of the PeA12K body Assembly is $780 \mathrm{gr}$, which is $25 \%$ less than that of the PeA12. Manufacturing of the polymer housing is less expensive, in comparison with PeA12. When developing a new model, it turned out to be expedient to study the influence of the body mass on the characteristics and position of natural frequencies in the operating range.

The AP2088 model $^{\dagger}$ has a number of differences from its predecessor, the PeA12. The body is structurally made of $20 \times 13$ steel. Among the electronics, a new generation piezoelectric sensing element and a two-channel preamp based on modern electronic components are used (the same output indicators with the PeA12 amplifier), figure 3.

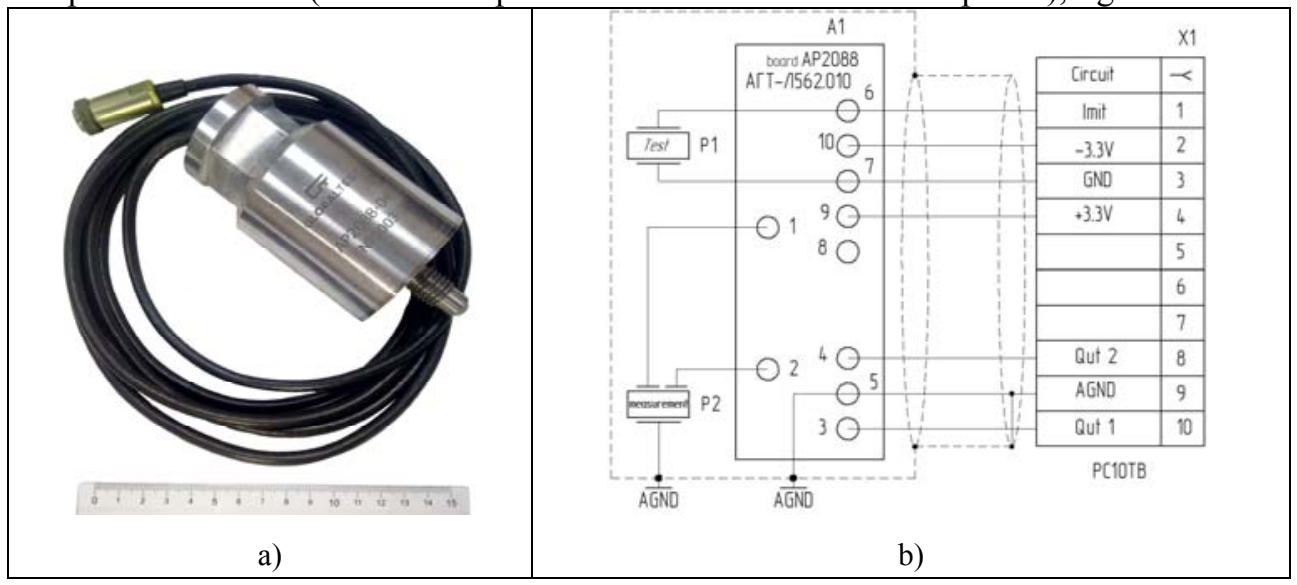

Fig. 3. Geophone AP2088: a) general view; b) schematic diagram of the converter AP2088

Modification of the electronic circuit allowed to reduce the output impedance; increase the dynamic range and power of the input signal; and provide simultaneous measurement of mechanical vibrations in two amplitude ranges. To reduce their own noise and eliminate interference between the two channels of amplifiers, a technical solution was chosen to implement a scheme with two independent channels on one piezoelectric element (different-sized silver deposition sectors on the surface of one piezoelectric element, three electrodes) and with a signal conversion sensitivity of $1.5 \mathrm{~V} / \mathrm{m}^{\circ} \mathrm{s}^{-2}$ and $48 \mathrm{~V} / \mathrm{m}^{\circ} \mathrm{s}^{-2}$, with a natural frequency in the range of $10-12 \mathrm{kHz}$. The case also provides the ability to send a test signal to a separate piezoelectric element, which allows you to remotely monitor the operational performance of the geophone.

The AP2088 model (compared to the PeA12) is 37\% lighter due to the possibility of using modern components of smaller sizes. The weight of the AP2088 body assembly is reduced from 1050 to $690 \mathrm{~g}$. The cable sheath is made of vibration-proof materials. The housing material is resistant to aggressive mine operating conditions, including explosive gas environments. In this model, it was possible to realize a uniform frequency response

\footnotetext{
† The AP2088 model was developed jointly with the employees of GlobalTest LLC
} 
$( \pm 10 \%)$ in a wide frequency range from 0.1 to $10 \mathrm{kHz}$ (at the level of $40 \mathrm{~dB} / \mathrm{V}$ ) and at the same time a high axial acceleration sensitivity of $10 \mathrm{~V} / \mathrm{m} \cdot \mathrm{s}^{-2}$ over the entire frequency range, figure 4 the frequency response resonances were shifted towards higher frequencies, in the range of 10-12 kHz, dynamic range of amplitude measurement up to $50 \mathrm{~dB}$.

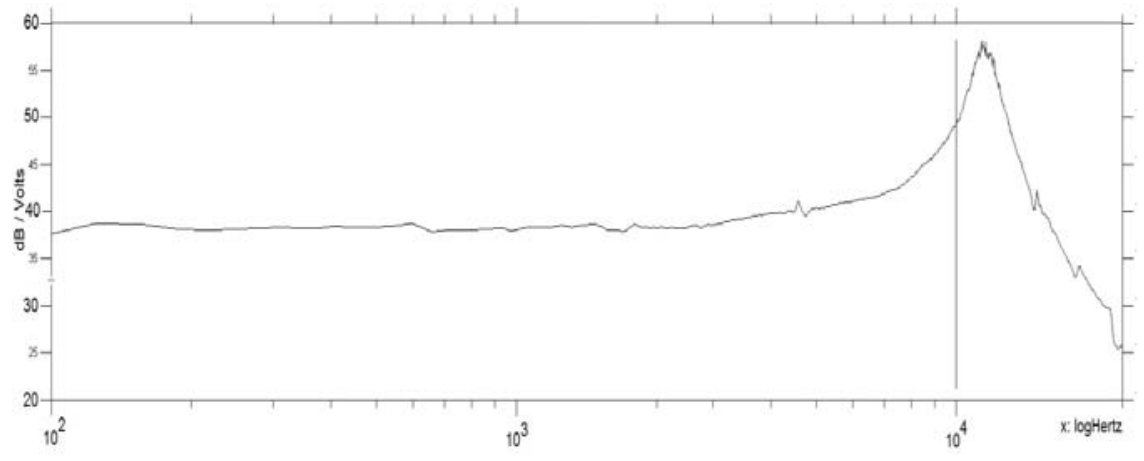

Fig. 4. Amplitude-frequency characteristics of the AP2088 geophone

The AP2088A model is completely based on the AP2088 electronic database. The hull structure was redesigned to be made from combined materials, including aircraft aluminium and 20X13 steel. The contact area is reduced in thickness and is also made of 20X13 steel. The external housing made of aircraft aluminium alloy is connected by an internal thread to the platform. The weight of the prototype AP2088A assembly is $570 \mathrm{gr}$, which is $46 \%$ less than the sensor PeA12. The enclosure is IP67 protected. The hull materials are resistant to aggressive mine operating conditions, including explosive gas environments.

\section{Results of researches}

Full-scale tests of new sensor models in the rockburst hazardous conditions were conducted at the Nikolaevsky underground mine of JSC MMC Dalpolymetall in 2019-2020. For prototypes of geophones, the following tasks were identified: to compare the parameters of acoustic emission signals and technological noise from different metrological characteristics of the geophones models; industrial testing and fault tolerance of new products in real-mode seismic and acoustic monitoring.

During the experiment, models of Pea12K, AP2088 and AP2088A sensors were placed at a distance of $0.5 \mathrm{~m}$ from each other in the faces of parallel wells $(100 \mathrm{~mm}$ in diameter, $2.5 \mathrm{~m}$ in length) drilled in the side of an underground mine near the current prototype of the PeA12 system "Prognoz-ADS".

The AP2088A model was added to the experiment later than the others. The conditions for recording signals and comparing their characteristics of the model were the same, included in a single digital channel, the same RADCi40 analog-to-digital converters, combined in a single network of seismic and acoustic monitoring of a rock mass, where a single time is maintained with an accuracy of $0.02 \mathrm{~ms}$.

During the tests, more than 1.2 million signals of different origin were registered, including: noise from mining machines and equipment, initiation of explosive charges, acoustic emission in the rock mass. The number of registered pulses differs for different sensor models. Thus, PeA12 registered $28 \%$ of the total number of signals in the frequency range from 0.1 to $9.3 \mathrm{kHz}, \mathrm{AP} 2088-48 \%$, PeA12K-24\%. Moreover, all the sensors registered dynamic manifestations of rock pressure (seismoacoustic events).

For analysis, the most important signals are seismic and acoustic events in the form of shocks from the depth of the rock mass and rockbursts. During the testing period in 2019, 9 
dynamic manifestations of rock pressure in the form of shocks were recorded (table 1), which were also visually registered by miners in underground mine workings.

Table 1. Seismoacoustic events, dynamic manifestations of rock pressure.

\begin{tabular}{|c|c|c|c|c|c|}
\hline \multirow{2}{*}{ Date } & \multirow{2}{*}{ Time } & \multicolumn{3}{|c|}{ Coordinate of the foci } & \multirow{2}{*}{ Energy, J } \\
\cline { 3 - 5 } & & $\mathbf{X}$ & $\mathbf{Y}$ & $\mathbf{Z}$ & \\
\hline \multirow{2}{*}{28.03 .2109} & $7: 21$ & 57654,9 & 77244,29 & $-486,14$ & 552,05 \\
\cline { 2 - 5 } & $7: 22$ & 57533,97 & 77322,03 & $-409,34$ & 1706,1 \\
\cline { 2 - 5 } & $7: 24$ & 57544,44 & 77249,53 & $-515,05$ & 4119,9 \\
\hline 01.07 .2019 & $9: 20$ & 57490,01 & 77312 & $-584,16$ & 86463,09 \\
\hline 15.08 .2019 & $7: 11$ & 57647,43 & 77294,42 & $-425,57$ & 10725,42 \\
\hline 10.10 .2019 & $11: 34$ & 57668,78 & 77283,58 & $-407,19$ & 4549,21 \\
\hline 16.10 .2019 & $12: 01$ & 57644,63 & 77234,26 & $-542,57$ & 37937,55 \\
\hline 17.11 .2019 & $9: 10$ & 57617,78 & 76917,85 & $-523,59$ & 14815 \\
\hline 11.01 .2020 & $12: 20$ & 57657,08 & 77205,21 & $-520,21$ & 19094,93 \\
\hline
\end{tabular}

The following characteristics and design parameters of registered signals that are important for monitoring impact hazard were analyzed and compared: signal amplitude in frequency periods $0.5-1,1-2, \ldots 9-10 \mathrm{kHz}$ (quantum); signal detection time; signal frequency $(\mathrm{kHz})$; signal duration (MS); MARSE* signal; the area of the signal (quantum). The signal amplitude in frequency periods is the most important parameter. If the sensor has a low sensitivity in a particular frequency range, it incorrectly registers the signal input, duration, area, or its calculated values (MARSE and acoustic emission pulse energy). The detection time of the signal ${ }^{\S}$ (the beginning of the signal) is an important parameter for locating acoustic emission sources and affects the accuracy of calculating the coordinates of the hearth. As the analysis showed, the time of signal detection in 4 out of 9 cases differs by 1-2 ms. The calculation of the coordinates of the signal source with the participation of AP2088 gave the smallest error (the square deviation of the coordinates of the focus on the location series with the participation of one of the prototypes varies from 1.6 to $4.3 \mathrm{~m}$ ).

The frequency of acoustic emission signals is calculated in the maximum value of the amplitude. As the analysis of the results showed, when registering signals with an amplitude of less than 10000 quantum, the frequency values for the models differ by 0.1 $0.5 \mathrm{kHz}$ in 4 of 9 cases, and in the rest by $\leq 1.5 \mathrm{kHz}$. When registering signals with amplitude of more than 10000 quantum, the frequencies differ by $1.0-2.5 \mathrm{kHz}$ according to the models. Analysis of the amplitude distribution over frequency ranges (Fourier transform) has shown that in some cases, when registering signals from 10000 quantum or more (the maximum possible value of the amplitude is 524256 quantum), the average amplitude reading in the frequency period of $4-5 \mathrm{kHz}$ is higher for the PeA12 and PeA12K sensors, and in other frequency periods - lower than for AP2088. The PeA12K model has $28 \%$ lower acceleration sensitivity than the PeA12. In the range of $4 . .5 \mathrm{kHz}$, the difference in readings between PeA12 and AP2088 reaches 133\%, (the maximum sensitivity of PeA12

\footnotetext{
$¥$ Measured Area of the Rectified Signal Envelope

$\S$ The automated system synchronizes the time of the recorders with an accuracy of up to $0.2 \mathrm{~ms}$
} 
sensors in the specified range, up to $7.5 \mathrm{~V} / \mathrm{m} \cdot \mathrm{s}^{-2}$ at the peak of resonance at $4.7 \mathrm{kHz}$ ), figure 2. When registering signals up to 10000 quantum, the amplitude value is always higher for the AP2088 sensor, figure 5. In some cases, the amplitude readings of the AP2088 differ several times (in the range of $3 \ldots 4 \mathrm{kHz}$ ), and in the range of $1 \ldots 2 \mathrm{kHz}$, the amplitude values of the AP2088 are greater by one order of magnitude. With all the differences in amplitudes for different geophones, the calculated value of the area and MARSE of the signal in all ranges is greater for the AP2088 geophone.
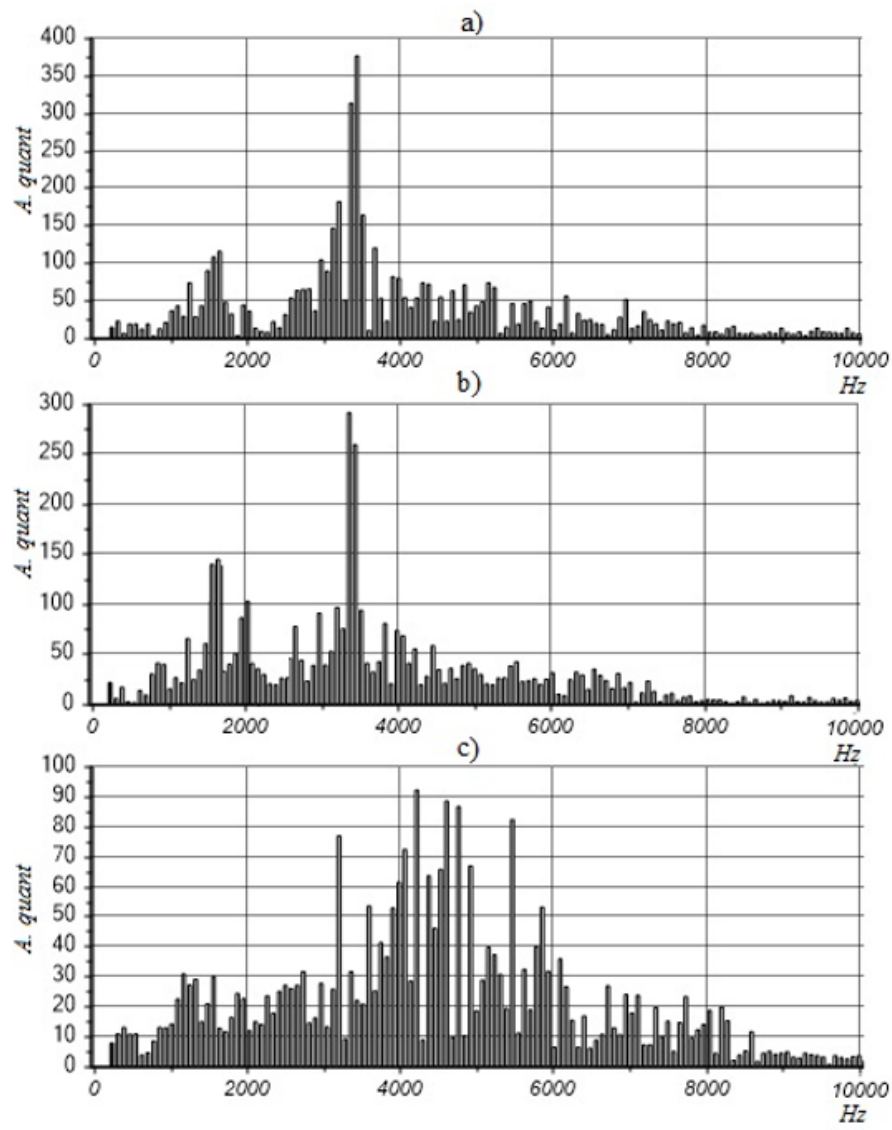

Fig. 5. Analysis of the amplitude distribution of the acoustic emission signal (Fourier transform method, where: a-signal AP2088, b-2088A, c-PeA12)

As a result of the experiment with newly developed models of geophones, updated characteristics of acoustic emission signals and calculated indicators of seismoacoustic events were obtained, which will further improve the reliability of the forecast of dangerous geodynamic phenomena and reduce the risk of man-made disasters at mining enterprises. At the end of industrial tests, the AP2088A model passed the necessary laboratory tests in the Federal state unitary enterprise "RFNC-VNIIEF" for inspection of the measuring instrument of the Russian Federation and was added to the register under the name AP2088 with the number 77757-20 dated 03.03.2020. Since 05.03.2020, the AP2088 transducers have been part of the "Prognoz-ADS" automated seismoacoustic system for monitoring the geomechanical state of the rock mass at the Rasvumchorrsky underground mine of the KB Apatit JSC. Installation of these systems as part of the AP2088 is planned at other underground mines that are dangerous for rockburst. 


\section{Conclusions}

1. The results of theoretical and experimental research have established the need to improve and modernize the high-sensitivity piezoelectric converters PeA12 of the resonant design of NPO Dalstandart, which have been successfully used for more than 30 years to locate sources of seismic and acoustic signals in the rock mass at a number of impact-prone mines. The main problem of the accuracy of measurement of acoustic signal parameters by the geophone PeA12 (and other models based on it) is due to the presence of several resonances in the operating frequency band.

2. The developed model of the AP2088 converter has successfully passed industrial testing as part of the automated monitoring system "Prognoz-ADS". During the test period (more than 1 year), seismoacoustic events were registered and verified in the rock mass, including rockbursts and shocks in the rock mass.

3. The use of highly sensitive $\left(\sim 10 \mathrm{~V} / \mathrm{m}^{\bullet} \mathrm{s}^{-2}\right)$ piezoacoustic converters AP2088 as part of the automated system "Prognoz-ADS" provides registration of acoustic emission in the rock mass in the frequency range from 0.1 to $10 \mathrm{kHz}$ with the energy of seismic events from 10 to $10^{6} \mathrm{~J}$, which will increase the reliability of the forecast of geodynamic phenomena and technogenic seismicity in the control zone of the system.

The reported study was funded by RFBR and NSFC according to the research project No. 20-55-53028.

\section{References}

1. Yu.I. Bolotin, A. Yu. Iskra, V. V. Nechaev (III all-union scientific and practical conference on acoustic emission: reports. Obninsk. Ch. 1. 1992)

2. Iskra, A. Yu. Systems for monitoring rock pressure (Moskow, IPKON an USSR, 1989)

3. V. V. Nechaev, A. Yu. Iskra, Yu. I. Bolotin. Proc. of the VII Intern. Con. on Surv. (1989)

4. Rasskazov I. Yu., Kalinov G. A., Iskra A. Yu., Kulikov D. A., Kharitonov K. O. Physical acoustics. Propagation and diffraction of waves. Geoacoustics: Proc. of the XVI session of the Russian acoustic society, 1 (2005)

5. I. Yu. Rasskazov, A. Yu. Iskra. Gorny Zhurnal, 1 (2007)

6. Rasskazov I. Yu., Gladyr A. V., Anikin P. A., Svyatetskiy V. S., Prosekin B. A. Gornyi Zhurnal, 8(2) (2013).

7. Anikin P. A., Rasskazov M. I., Migunov D. S., Tereshkin A. A., Gladyr A.V., Tsoi D.

I., Lomov M. A. Materials of the VIII International scientific and practical conference (2017)

8. Fedyanin, A.S., Rasskazov, I.U., Anikin, P.A. Proc. 12th Conference and Exhibition Engineering Geophysics (2016)

9. I.Ju. Rasskazov, B.G. Saksin, M.I. Potapchuk \& P.A. Anikin. Proc. of the European rock mechanics symposium, 1 (2018)

10. Pavel Anikin, Iuliia Fedotova, Gennady Kursakin and Mikhail Lomov. E3S Web of Conferences, 129 (2019) 